\title{
PRIME IDEALS IN STRUCTURAL MATRIX NEAR-RINGS
}

\author{
ENOCH K. S. LEE
}

\begin{abstract}
This paper studies different types of prime ideals and their radicals in structural matrix near-rings. Relationships between various types of prime ideals of a near-ring and the corresponding structural matrix near-ring are given.
\end{abstract}

\section{Introduction}

Matrix near-rings were first studied by Heatherly [5] and Ligh [6] in the context of distributive and n-distributive near-rings. In 1986, Meldrum and van der Walt [9] defined a matrix near-ring over a near-ring as a subnear-ring of a transformation nearring. Certain prime ideals and their associated radicals have been studied since then. (See Booth and Groenewald [1] and Groenewald [4].) Furthermore, van der Walt and van Wyk [11] initiated the study of structural martix near-rings (see also [12]). A structural matrix near-ring " $\mathcal{M}_{n}(B, R)$ ", which depends virtually on the structure of the Boolean matrix " $B$ ", is a subnear-ring of the matrix near-ring " $\mathcal{M}_{n}(R)$ ". In this paper, we study several types of prime ideals and their radicals in structural matrix near-rings over a near-ring with identity. We also provide a different way of describing the set $\left(R^{n}(j, L): R^{n}(j, R)\right)$, which was studied in [11], [12], and [13]. For basic properties concerning near-rings and matrix near-rings, we refer to [3], [7], [9], and [10].

\section{Preliminaries}

Throughout this paper, a near-ring will be a right zero-symmetric near-ring with identity, while a subnear-ring will always be a subnear-ring with the identity. By an ideal of a near-ring we will mean a two-sided ideal. $R$ will be a generic symbol for a near-ring (except where noted). Let $n$ be a fixed natural number and let $\underline{n}$ denote the set $\{1,2, \ldots, n\} . R^{n}$ will denote the direct sum of $n$ copies of $(R,+)$, and elements of $R^{n}$ will

Received September 3, 1993; revised January 24, 1994.

1991 Mathematics Subject Classification. 16Y30.

Key words and phrases. Near-rings, prime ideals, matrix near-rings. 
be represented by $\bar{u}, \bar{v}$, etc., and considered as $n$-tuples, for instance, $\bar{u}=\left(u_{1}, \cdots, u_{n}\right)$. If $\bar{u}, \bar{v} \in R^{n}$, we define the product $\bar{u} \bar{v}=\left(u_{1} v_{1}, \cdots, u_{n} v_{n}\right)$; if also $a \in R$, let $\bar{u} a=$ $\left(u_{1} a, \cdots, u_{n} a\right)$. Denote the $n$-tuple with 1 in the $i$-th component and 0 elsewhere by $\bar{\epsilon}_{i}$.

Recall that $\mathcal{M}_{0}(G)$ is the near-ring of all zero-preserving mappings of $(G,+)$ into itself for any group $(G,+)$. The mappings $\iota_{j}$ and $\pi_{j}$ will denote the $j$-th coordinate injection and projection, respectively. That is $\iota_{j}(u)=(0, \cdots, u, \cdots, 0)$ where $u$ is in the $j$-th position and $\pi_{j}\left(u_{1}, \ldots, u_{n}\right)=u_{j}$. For $r \in R$, let $f^{r} \in M_{0}(R)$ be defined by $f^{r}(s)=r s$ for all $s \in R$. We define $f_{i j}^{r}: R^{n} \rightarrow R^{n}$ where $f_{i j}^{r}=\iota_{i} f^{r} \pi_{j}$ for $i, j \in \underline{n}$ and $r \in R$.

Definition 2.1 [9] The subnear-ring of $M_{0}\left(R^{n}\right)$ generated by the set $\left\{f_{i j}^{r} \mid r \in\right.$ $R ; i, j \in \underline{n}$ \} is called an $n \times n$ matrix near-ring over $R$, denoted by $\mathcal{M}_{n}(R)$, and each element of $\mathcal{M}_{n}(R)$ is called a matrix.

An $n \times n$ matrix $B=\left[b_{i j}\right]$ will be a generic symbol for a Boolean matrix of size $n$, i.e., $b_{i j}=0$ or 1 for $i, j \in \underline{n}$. We shall henceforth assume the Boolean matrix $B$ satisfies the conditions: (1) $b_{i i}=1$ for each $i \in \underline{n}$; and (2) if $b_{i j}=b_{j k}=1$, then $b_{i k}=1$. Let $\bar{u}, \bar{v} \in R^{n}$. We wirte:

$$
\bar{u} \sim_{i} \bar{v} \text { if and only if } \pi_{j} \bar{u}=\pi_{j} \bar{v} \text { for all } j \text { such that } b_{i j}=1 \text {. }
$$

Remark. If $b_{i j}=0$, then $\bar{\epsilon}_{j} \sim_{i} \overline{0}$ where $\overline{0}=(0, \cdots, 0)$.

Definition 2.2. [11] Let $\mathcal{M}_{n}(B, R)=\left\{X \in \mathcal{M}_{n}(R) \mid\left(\forall_{i} \in \underline{n}, \forall \bar{u}, \bar{v} \in R^{n}\right)\left(\bar{u} \sim_{i}\right.\right.$ $\left.\left.\bar{v} \Rightarrow \pi_{i} X \bar{u}=\pi_{i} X \bar{v}\right)\right\}$. We call $\mathcal{M}_{n}(B, R)$ the $n \times n$ structural matrix near-ring over $R$ with respect to $B$.

Van der Walt and van Wyk [11] showed that $\mathcal{M}_{n}(B, R)$ is also the subnear-ring of $\mathcal{M}_{n}(R)$ generated by the set $\left\{f_{i j}^{r} \mid r \in R\right.$ and $\left.b_{i j}=1\right\}$. In view of this result, we can now introduce the concept of representations of structural matrices. Let $\mathbb{E}_{n}(B, R)$ be the subset of words over the alphabet of symbols $\left\{f_{i j}^{r} \mid r \in R\right.$ and $\left.b_{i j}=1\right\} \cup\{(),+$,$\} ,$ recursively defined by the following rules:

(1) $f_{i j}^{r} \in \mathbb{E}_{n}(B, R)$ for all $r \in R$ and for $i, j \in \underline{n}$ with $b_{i j}=1$;

(2) if $\mathbf{X}$ and $\mathbf{Y} \in \mathbb{E}_{n}(B, R)$, then $\mathbf{X}+\mathbb{Y} \in \mathbb{E}_{n}(B, R)$;

(3) if $\mathbf{X}$ and $\mathbb{Y} \in \mathbb{E}_{n}(B, R)$, then $(\mathbf{X})(\mathbb{Y}) \in \mathbb{E}_{n}(B, R)$.

The length of an element $\mathbf{X}$ of $\mathbb{E}_{n}(B, R)$ is defined as the number of $f_{i j}^{r}$ in $\mathbf{X}$ such that $b_{i j}=1$. (Note: there might be more than one expression for any matrices. The "length" is defined on expressions of matrices, not on matrices.) The weight, $\omega(A)$, of a matrix $A$ of $\mathcal{M}_{n}(B, R)$, is the length of an expression in $\mathbb{E}_{n}(B, R)$ of minimal length representing $A$. Observe that for any matrix $A$ of $\mathcal{M}_{n}(B, R)$ with $1<\omega(A)$, there exist matrices $C, D \in \mathcal{M}_{n}(B, R)$ of minimal length representing $A$. Observe that for any matrix $A$ of $\mathcal{M}_{n}(B, R)$ with $1 \leq \omega(C), \omega(D)<\omega(A)$ such that $A=C+D$ or $C D$. 
The following results will be useful throughout this paper.

Lemma 2.3.

(1) [Lemma 2.1,1] If $X \in \mathcal{M}_{n}(R), s \in R$ and $\bar{u} \in R^{n}$, then $X(\bar{u} s)=(X \bar{u}) s$.

(2) [Proposition 2.2,11] Let $X \in \mathcal{M}_{n}(B, R)$. For any $\bar{u}$ and $\bar{v} \in R^{n}$, if $\bar{u} \sim_{i} \bar{v}$, then $X \bar{u} \sim_{i} X \bar{v}$.

(3) $\left[\right.$ Lemma 2.3,11] Let $i, j \in \underline{n}$. Then $b_{i j}=1$ if and only if $f_{i j}^{r} \in \mathcal{M}_{n}(B, R)$ for all $r \in R$.

Definition 2.4. Let $\mathcal{L} \subseteq \mathcal{M}_{n}(B, R)$ and $j \in \underline{n}$. Then:

(1) $\prod_{B}(R, j)=\left\{\left(u_{1}, \cdots, u_{n}\right) \in R^{n} \mid u_{i}=0\right.$ if $\left.b_{i j}=0\right\}$.

(2) $\mathcal{L}_{(j)}[B]=\left\{x \in R \mid(\exists X \in \mathcal{L})\left(\exists \bar{u} \in \prod_{B}(R, j)\right)\left(x=\pi_{j} X \bar{u}\right)\right\}$.

If there is no ambiguity, we write $\Pi(R, j)$ and $\mathcal{L}_{(j)}$ for $\prod_{B}(R, j)$ and $\mathcal{L}_{(j)}[B]$, respectively.

Remark.

(1) If $X \in \mathcal{M}_{n}(B, R)$ and $i, j \in \underline{n}$, then $f_{i i}^{1} X f_{j j}^{1}=f_{i j}^{x}$ where $x=\pi_{i} X \bar{\epsilon}_{j}$.

(2) $\left(\mathcal{M}_{n}(B, R)\right)_{(i)}=R$ for $i \in \underline{n}$.

Suppose $H$ is a subset of $R$ such that $R H \subseteq H$. Then we say $H$ is a left invariant subset. Right invariant and two-sided invariant subsets can be defined in a similar way.

\section{Lemma 2.5.}

(1) Let $\bar{u}=\left(u_{1}, \cdots, u_{n}\right) \in \prod_{B}(R, j)$. Then $\bar{u}=\left(\sum_{i=1}^{n} f_{i j}^{u_{i}}\right) \bar{\epsilon}_{j}$.

(2) Let $\mathcal{Y}$ be a right invariant subset of $\mathcal{M}_{n}(B, R)$ and $j \in \underline{n}$. If $X \in \mathcal{Y}$ and $\bar{u} \in \Pi_{B}(R, j)$, then there is $Y \in \mathcal{Y}$ such that $X \bar{u}=Y \bar{\epsilon}_{j}$.

Proof. Since $\left(u_{1}, \cdots, u_{n}\right) \in \Pi(R, j)$, if $b_{i j}=0$ then $u_{i}=0$. Therefore we have part (1). Part (2) follows immediately from part (1) and the fact that $\bar{\epsilon}_{j} \in \Pi(R, j)$.

Proposition 2.6. Let $\mathcal{L}$ be a two-sided invariant subset of $\mathcal{M}_{n}(B, R)$ and $i \in \underline{n}$. Then $x \in \mathcal{L}_{(i)}$ if and only if $f_{i i}^{x} \in \mathcal{L}$. Moreover, if also $b_{i j}=b_{j i}=1$, then $\mathcal{L}_{(i)}=\mathcal{L}_{(j)}$.

Proof. Note that $x \in \mathcal{L}_{(i)}$ if and only if $x=\pi_{i} X \bar{u}$ for some $X \in \mathcal{L}$ and $\bar{u} \in \Pi(R, i)$. By Lemma 2.5(2), there is a $Y \in \mathcal{L}$ such that $X \bar{u}=Y \bar{\epsilon}_{i}$ and so $x=\pi_{i} Y \bar{\epsilon}_{i}$. Thus $f_{i i}^{x}=f_{i i}^{1} Y f_{i i}^{1} \in \mathcal{L}$. Conversely, from the fact that $\bar{\epsilon}_{i} \in \Pi(R, i)$ and $x=\pi_{i} f_{i i}^{x} \bar{\epsilon}_{i}$, we have: if $f_{i i}^{x} \in \mathcal{L}$, then $x \in \mathcal{L}_{(i)}$.

Suppose also that $b_{i j}=b_{j i}=1$. Since $f_{i i}^{x} \in \mathcal{L}$ if and only if $f_{j j}^{x}=f_{j i}^{1} f_{i i}^{x} f_{i j}^{1} \in \mathcal{L}$, we then have the desired result.

Proposition 2.7. Let $\mathcal{L}$ be an ideal of $\mathcal{M}_{n}(B, R)$. Then $\mathcal{L}_{(i)}$ is an ideal of $R$ for any $i \in \underline{n}$. 
Proof. Suppose $x, y \in \mathcal{L}_{(i)}$ and $r, s \in R$. We have $f_{i i}^{x-y}=f_{i i}^{x}-f_{i i}^{y}$ is in $\mathcal{L}$. Hence $x-y$ is in $\mathcal{L}_{(i)}$. Using a similar argument, we can show $r+x-r, x r, s(x+r)-s r$ are in $\mathcal{L}_{(i)}$. Therefore $\mathcal{L}_{(i)}$ is an ideal of $R$.

Observe that whenever $\mathcal{L}$ is an ideal of $\mathcal{M}_{n}(B, R)$, we have: $\mathcal{L}$ is a proper ideal if and only if $\mathcal{L}_{(i)}$ is a proper ideal for some $i \in \underline{n}$.

Proposition 2.8. If $j \in \underline{n}$ and $X \in \mathcal{M}_{n}(B, R)$, then $X\left(\prod_{B}(R, j)\right) \subseteq \prod_{B}(R, j)$.

Proof. In view of Lemma 2.5, we note that it suffices to show that $\pi_{i} X \bar{\epsilon}_{j}=0$ whenever $X \in \mathcal{M}_{n}(B, R)$ and $b_{i j}=0$. But this follows immediately from the fact that $\bar{\epsilon}_{j} \sim_{i} \overline{0}$ whenever $b_{i j}=0$. (See the remark after Definition 2.1.)

Definition 2.9. Let $i \in \underline{n}$ and $L \subseteq R$. Then:

(1) $\amalg_{B}(i, L)=\left\{\left(u_{1}, \cdots, u_{n}\right) \in R^{n} \mid u_{j} \in L\right.$ if $\left.b_{i j}=1\right\}$;

(2) $L^{(i)}[B]=\left\{X \in \mathcal{M}_{n}(B, R) \mid X\left(\prod_{B}(R, i)\right) \subseteq \amalg_{B}(i, L)\right\}$.

If no confusion can occur, we write $\amalg(i, L)$ and $L^{(i)}$ for $\amalg_{B}(i, L)$ and $L^{(i)}[B]$, respectively.

Remark.

(1) Let $X \in \mathcal{M}_{n}(B, R)$ and $L \subseteq R$. We have $X \in L^{(i)}$ if and only if $\pi_{j} X \bar{u} \in L$ whenever $\bar{u} \in \prod(R, i)$ and $b_{i j}=1$.

(2) $R^{(i)}=\mathcal{M}_{n}(B, R)$ for any $i \in \underline{n}$.

Lemma 2.10. let $L$ be a left ideal of $R$ and $i \in \underline{n}$. Then $\amalg_{B}(i, L)$ is an $\mathcal{M}_{n}(B, R)$-ideal of $R^{n}$. (Here we consider $R^{n}$ as an $\mathcal{M}_{n}(B, R)$-module.)

Proof. Obviously $\amalg(i, L)$ is a normal subgroup of $R^{n}$. We prove $X(\bar{u}+\bar{v})-X \bar{v}$ is in $\amalg(i, L)$ for $\bar{u} \in \amalg(i, L), \bar{v} \in R^{n}, X \in \mathcal{M}_{n}(B, R)$ by means of induction on the weights of matrices. In fact, it suffices to show $\pi_{j}(X(\bar{u}+\bar{v})-X \bar{v}) \in L$ for all $j$ such that $b_{i j}=1$. So assume $b_{i j}=1$. If $\omega(X)=1$, then $X=f_{h k}^{r}$ with $b_{h k}=1$. We have:

$$
\pi_{j}\left(f_{h k}^{r}(\bar{u}+\bar{v})-f_{h k}^{r} \bar{v}\right)= \begin{cases}0 & \text { if } j \neq h, \\ r\left(u_{k}+v_{k}\right)-r v_{k} & \text { if } j=h .\end{cases}
$$

If $j=h$, then $b_{i k}=b_{i j} b_{j k}=1$ and hence $u_{k} \in L$. Therefore we have $\pi_{j}\left(f_{h k}^{r}(\bar{u}+\right.$ $\left.\bar{v})-f_{h k}^{r} \bar{v}\right) \in L$. For purposes of induction, we assume $X(\bar{u}+\bar{v})-X \bar{v}$ is in $\amalg(i, L)$ for $X \in \mathcal{M}_{n}(B, R)$ with $1 \leq \omega(X) \leq m$. Now if $\omega(X)=m+1$, then there are $C, D \in \mathcal{M}_{n}(B, R)$ with $1 \leq \omega(C), \omega(D) \leq m$ such that either $X=C+D$ or $C D$. In the first case we have $X(\bar{u}+\bar{v})-X \bar{v}=C(\bar{u}+\bar{v})+(D(\bar{u}+\bar{v})-D \bar{v})-C \bar{v}$ is in $\amalg(i, L)$. In the second case we have $X(\bar{u}+\bar{v})-X \bar{v}=C((D(\bar{u}+\bar{v})-D \bar{v})+D \bar{v})-C D \bar{v}$ is in $\amalg(i, L)$. By the principle of induction, we have that $\amalg(i, L)$ is an $\mathcal{M}_{n}(B, R)$-ideal of $R^{n}$.

Observe that if we assume that $L$ is a left $R$-subgroup in the above lemma, then we can show that $X\left(\coprod_{B}(i, L)\right) \subseteq \amalg_{B}(i, L)$ for $X \in \mathcal{M}_{n}(B, R)$.

Proposition 2.11. 
(1) If $L$ is an ideal of $R$, then $L^{(i)}$ is an ideal of $\mathcal{M}_{n}(B, R)$ for $i \in \underline{n}$.

(2) If $L$ is a left $R$-subgroup, then $L^{(i)}$ is a two-sided $\mathcal{M}_{n}(B, R)$-subgroup for $i \in \underline{n}$.

Proof. Proposition 2.8 and Lemma 2.10 give part (1). Use proposition 2.8 and the observation after Lemma 2.10 to obtain part (2).

Lemma 2.12.

(1) Let $L$ be a right invariant subset of $R$. Then $f_{i i}^{x} \in L^{(i)}$ if and only if $x \in L$.

(2) Let $L$ be a proper two-sided R-subgroup and $b_{k h}=1$. Then $f_{k h}^{1} \in L^{(i)}$ if and only if $b_{i k}=0$ or $b_{h i}=0$.

Proof.

(1) Suppose $f_{i i}^{x} \in L^{(i)}$. Since $\bar{\epsilon}_{i} \in \Pi(R, i)$, we have $f_{i i}^{x} \bar{\epsilon}_{i} \in \amalg(i, L)$. In particular, $x=\pi_{i} f_{i i}^{x_{i}} \bar{c}_{i} \in L$. Suppose now $x \in L, \bar{u}=\left(u_{1}, \cdots, u_{n}\right) \in \Pi(R, i)$, and $b_{i h}=1$.

Then:

$$
\pi_{h} f_{i i}^{x} \bar{u}= \begin{cases}0 & \text { if } h \neq i, \\ x u_{i} & \text { if } h=i .\end{cases}
$$

So $\pi_{h} f_{i i}^{x} \bar{u} \in L$. This yields $f_{i i}^{x} \bar{u} \in \amalg(i, L)$ and hence $f_{i i}^{x} \in L^{(i)}$.

(2) Assume $f_{k h}^{1} \in L^{(i)}$. For purposes of contradiction, suppose $b_{i k}=b_{h i}=1$. Thus $f_{i k}^{1}$, $f_{h i}^{1} \in \mathcal{M}_{n}(B, R)$. This implies $f_{i i}^{1}=f_{i k}^{1} f_{k h}^{1} f_{h i}^{1} \in L^{(i)} ;$ hence $1 \in L$ from part (1). So $L$ is not proper. This proves the result one way. Conversely, assume $b_{i k}=0$ or $b_{h i}=0$. Let $\bar{u} \in \Pi(R, i)$ and $b_{i m}=1$. Then:

$$
\pi_{m} f_{k h}^{1} \bar{u}= \begin{cases}0 & \text { if } m \neq k \\ u_{h} & \text { if } m=k .\end{cases}
$$

If $m=k$, then $b_{i k}=1$. Thus from the assumption, we have $b_{h i}=0$. Since $\bar{u} \in \Pi(R, i)$, we have $u_{h}=0$. (See Definition 2.4.) Then $\pi_{m} f_{k h}^{1} \bar{u}=0 \in L$. This yields $f_{k h}^{1} \bar{u} \in \amalg(i, L)$. Hence $f_{k h}^{1} \in L^{(i)}$.

\section{Proposition 2.13 .}

(1) Let $L$ be a proper two-sided $R$-subgroup and $k \in \underline{n}$. Then we have:

$$
\left(L^{(i)}\right)_{(k)}= \begin{cases}R & \text { if } b_{i k}=0 \text { or } b_{k i}=0 \\ L & \text { if } b_{i k}=b_{k i}=1\end{cases}
$$

(2) Let $\mathcal{L}$ be a left invariant subset of $\mathcal{M}_{n}(B, R)$. Then $\mathcal{L} \subseteq\left(\mathcal{L}_{(j)}\right)^{(j)}$ for $j \in \underline{n}$. Proof.

(1) Note that $L^{(i)}$ and $\left(L^{(i)}\right)_{(k)}$ are two-sided $R$-subgroups. (See Propositions 2.11(2) and 2.6.) Suppose $b_{i k}=0$ or $b_{k i}=0$. Lemma 2.12(2) gives $f_{k k}^{1} \in L^{(i)}$. From Proposition 2.6, we have $1 \in\left(L^{(i)}\right)_{(k)}$. Therefore $\left(L^{(i)}\right)_{(k)}=R$. Suppose now $b_{i k}=b_{k i}=1$. Proposition 2.6 implies $\left(L^{(i)}\right)_{(i)}=\left(L^{(i)}\right)_{(k)}$. Note $x \in\left(L^{(i)}\right)_{(i)}$ if and only if $f_{i i}^{x} \in L^{(i)}$ if and only if $x \in L$. Hence $\left(L^{(i)}\right)_{(k)}=L$. 
(2) Observe that if $\bar{u} \in \Pi(R, j)$ and $A \in \mathcal{L}$, then $\pi_{j} A \bar{u} \in \mathcal{L}_{(j)}$. So if $b_{j i}=1$ and $X \in \mathcal{L}$, then $f_{j i}^{1} \in \mathcal{M}_{n}(B, R)$ and hence $f_{j i}^{1} X \in \mathcal{L}$ for $X \in \mathcal{L}$. This implies that whenever $\bar{u} \in \Pi(R, j)$ we have $\pi_{i} X \bar{u}=\pi_{j} f_{j i}^{1} X \bar{u} \in \mathcal{L}_{(j)}$. This yields $X \bar{u} \in \coprod\left(j, \mathcal{L}_{(j)}\right)$. Hence $X \in\left(\mathcal{L}_{(j)}\right)^{(j)}$.

\section{Lemma 2.14.}

(1) Let $U \subseteq V \subseteq R$. Then $U^{(i)} \subseteq V^{(i)}$ for $i \in \underline{n}$.

(2) Let $\mathcal{L} \subseteq \mathcal{K} \subseteq \mathcal{M}_{n}(B, R)$. Then $\mathcal{L}_{(i)} \subseteq \mathcal{K}_{(i)}$ for $i \in \underline{n}$.

(3) Let $\Gamma$ be a collection of subsets of $R$. Then $\left(\cap_{U \in \Gamma} U\right)^{(i)}=\cap_{U \in \Gamma} U^{(i)}$ for $i \in \underline{n}$.

(4) Let $\Omega$ be a collection of two-sided invariant subsets of $\mathcal{M}_{n}(B, R)$. Then $\left(\cap_{\mathcal{L} \in \Omega} \mathcal{L}\right)_{(i)}=\cap_{\mathcal{L} \in \Omega} \mathcal{L}_{(i)}$ for $i \in \underline{n}$. Furthermore, if $\mathcal{L}, \mathcal{K} \in \Omega$, then $\mathcal{L}_{(i)} \mathcal{K}_{(i)} \subseteq(\mathcal{L K})_{(i)}$ for $i \in \underline{n}$.

Proof. Parts (1), (2), and (3) follow immediately from definitions. We only show part (4). Use Proposition 2.6 to obtain that $x \in\left(\cap_{\mathcal{L} \in \Omega} \mathcal{L}\right)_{(i)}$ if and only if $f_{i i}^{x} \in \cap_{\mathcal{L} \in \Omega} \mathcal{L}$ if and only if $x \in \mathcal{L}_{(i)}$ for $\mathcal{L} \in \Omega$. Thus we have $\left(\cap_{\mathcal{L} \in \Omega} \mathcal{L}\right)_{(i)}=\cap_{\mathcal{L} \in \Omega} \mathcal{L}_{(i)}$. Furthermore, suppose $\mathcal{L}, \mathcal{K} \in \Omega$. If $x \in \mathcal{L}_{(i)}$ and $y \in \mathcal{K}_{(i)}$, then $f_{i i}^{x} \in \mathcal{L}$ and $f_{i i}^{y} \in \mathcal{K}$. Since $f_{i i}^{x y}=$ $f_{i i}^{x} f_{i i}^{y} \in \mathcal{L K}$, we have $x y \in(\mathcal{L K})_{(i)}$.

Lemma 2.15. Let $L$ and $H$ be proper two-sided $R$-subgroups. Then $L^{(i)}=$ $H^{(j)}$ if and only if $L=H$ and $b_{i j}=b_{j i}=1$.

Proof. Suppose $L=H$ and $b_{i j}=b_{j i}=1$. Observe that $\Pi(R, i)=\prod(R, j)$ and $\amalg(i, L)=\amalg(j, L)$ will suffice to show $L^{(i)}=H^{(j)}$. If $\bar{u} \in \Pi(R, i)$ and $b_{k j}=0$, then $b_{k i}=0$ and so $\pi_{k} \bar{u}=0$. Thus $\bar{u} \in \Pi(R, j)$ and hence $\Pi(R, i) \subseteq \Pi(R, j)$. Similarly, we have $\Pi(R, j) \subseteq \Pi(R, i)$. Therefore $\Pi(R, i)=\Pi(R, j)$. If $\bar{v} \in \coprod(i, L)$ and $b_{j k}=1$, then $b_{i k}=1$ and so $\pi_{k} \bar{v} \in L$. Thus $\bar{v} \in \coprod(j, L)$. This gives $\amalg(i, L) \subseteq \amalg(j, L)$. We then have $\amalg(i, L)=\amalg(j, L)$. This proves the result one way. Conversely, suppose $L^{(i)}=H^{(j)}$. From Proposition 2.13, we have:

$$
L=\left(L^{(i)}\right)_{(i)}=\left(H^{(j)}\right)_{(i)}= \begin{cases}N & \text { if } b_{i j}=0 \text { or } b_{j i}=0 \\ H & \text { if } b_{i j}=b_{j i}=1\end{cases}
$$

This yields $L=H$ and $b_{i j}=b_{j i}=1$.

\section{Prime Ideals And Radicals}

Recall that a proper ideal $P$ of $R$ is called

(1) a prime ideal if for any ideals $U$ and $V$ of $R$ such that $(U V \subseteq P) \Rightarrow(U \subseteq P)$ or $(V \subseteq P)$.

(2) a 1-prime ideal if for any $a, b \in R$ such that $(a R b \subseteq P) \Rightarrow(a \in P)$ or $(b \in P)$.

(3) an equiprime ideal if for any $a \in R \backslash P, x$ and $y \in R$ such that $(\forall r \in R$,arx - ary $\in$ $P) \Rightarrow(x-y) \in P$. (See [1] and [2].) 
(4) a completely prime ideal if for any $a, b \in R$ such that $(a b \in P) \Rightarrow(a \in P)$ or $(b \in P)$.

We write $\mathbb{P}_{\nu}(R)$ and $\operatorname{Spec}_{\nu}(R)$ for the intersection and the collection of all proper prime, 1-prime, equiprime, or completely prime ideals of $R$ according to whether $\nu=$ $0,1, e$, or 2 .

Theorem 3.1. Let $P$ be a prime (resp. 1-prime, equiprime) ideal of $R$. Then $P^{(i)}$ is a prime (resp. 1-prime, equiprime) ideal of $\mathcal{M}_{n}(B, R)$ for $i \in \underline{n}$.

Proof. Let $P$ be a prime ideal of $R$ and $\mathcal{U}, \mathcal{V}$ ideals of $\mathcal{M}_{n}(B, R)$ such that $\mathcal{U V} \subseteq P^{(i)}$. We want to show $\mathcal{U} \subseteq P^{(i)}$ or $\mathcal{V} \subseteq P^{(i)}$. Use Proposition 2.13 and Lemma 2.14 to obtain the following sequentially: $(\mathcal{U V})_{(i)} \subseteq\left(P^{(i)}\right)_{(i)}, \mathcal{U}_{(i)} \mathcal{V}_{(i)} \subseteq P, \mathcal{U}_{(i)} \subseteq P$ or $\mathcal{V}_{(i)} \subseteq P$, and $\mathcal{U} \subseteq P^{(i)}$ or $\mathcal{V} \subseteq P^{(i)}$. Thus we are done. Now suppose $P$ is a 1 prime ideal of $R$ and $X, Y \in \mathcal{M}_{n}(B, R)$ such that $X, Y \notin P^{(i)}$. We want to show $X \mathcal{M}_{n}(B, R) Y \& P^{(i)}$. From definitions, there are $\bar{u}, \bar{v} \in \Pi(R, i)$ and $h, k \in \underline{n}$ with $b_{i h}=b_{i k}=1$ such that $a=\pi_{h} X \bar{u} \notin P$ and $b=\pi_{k} Y \bar{v} \notin P$. Therefore there exists $r \in R$ such that $a r b \notin P$. Since $\pi_{h}((X \bar{u}) r b)=\left(\pi_{h} X \bar{u}\right) r b=a r b \notin P$, we have $(X \bar{u}) r b \notin$ $\coprod(i, P)$. Furthermore, $(X \bar{u}) r b=X(\bar{u} r b)$ by Lemma $2.3(1)$, so $X(\bar{u} r b) \notin \amalg(i, P)$. Observe that $\bar{u} r b=\left(u_{1} r, \cdots, u_{n} r\right)\left(\pi_{k} Y \bar{v}\right)=\left(\sum_{j=1}^{n} f_{j k}^{u_{j} r}\right) Y \bar{v}$ where $\bar{u}=\left(u_{1}, \cdots, u_{n}\right)$. Since $X\left(\sum_{j=1}^{n} f_{j k}^{u_{j} r}\right) Y \bar{v}=X(\bar{u} r b) \notin \amalg(i, P)$, we have $X \mathcal{M}_{n}(B, R) Y \nsubseteq P^{(i)}$. Hence $P^{(i)}$ is 1-prime. The proof of the equiprime case is similar to that of the 1-prime case. (See also [Proposition 2.2, 1].)

Theorem 3.2. Let $\mathcal{Q}$ be a 1-prime (resp. equiprime, completely prime) ideal of $\mathcal{M}_{n}(B, R)$. Then $\mathcal{Q}_{(i)}$ is a 1-prime (resp. equiprime, completely prime) ideal of $R$ for $i \in \underline{n}$.

Proof. We will prove the 1-prime case. Suppose $\mathcal{Q}$ is a 1-prime ideal of $\mathcal{M}_{n}(B, R)$. Let $a$ and $b \in R$ such that $a R b \subseteq \mathcal{Q}_{(i)}$. Then $f_{i i}^{a r b} \in \mathcal{Q}$ for all $r \in R$. Now if $X \in$ $\mathcal{M}_{n}(B, R)$ and $x=\pi_{i} X \bar{\epsilon}_{i}$ then $f_{i i}^{a} X f_{i i}^{b}=f_{i i}^{a x b} \in \mathcal{Q}$. This implies $f_{i i}^{a} \in \mathcal{Q}$ or $f_{i i}^{b} \in \mathcal{Q}$. Hence $a \in \mathcal{Q}_{(i)}$ or $b \in \mathcal{Q}_{(i)}$. Similarly, we can prove the equiprime and completely prime cases.

Lemma 3.3. Let $\mathcal{Q}$ be an ideal of $\mathcal{M}_{n}(B, R)$ and $A \in \mathcal{M}_{n}(B, R)$. Then the following are equivalent:

(1) $A \in\left(\mathcal{Q}_{(i)}\right)^{(i)}$;

(2) If $\bar{u} \in \prod(R, i)$ and $b_{i h}=1$, then $\pi_{h} A \bar{u} \in \mathcal{Q}_{(i)}$;

(3) If $\bar{u} \in \prod(R, i)$ and $b_{i h}=1$, then $f_{i i}^{a_{h}} \in \mathcal{Q}$ where $a_{h}=\pi_{h} A \bar{u}$.

Proof. The equivalence of (1) and (2) follows directly from Definitions 2.4 and 2.9. The equivalence of (2) and (3) is obtained by using Proposition 2.6.

Theorem 3.4. Let $\mathcal{Q}$ be a 1-prime ideal of $\mathcal{M}_{n}(B, R)$. Then there exists a $k \in \underline{n}$ such that $\left(\mathcal{Q}_{(k)}\right)^{(k)}=\mathcal{Q}$. Hence $\cap_{i=1}^{n}\left(\mathcal{Q}_{(i)}\right)^{(i)}=\mathcal{Q}$. 
Proof. We have shown that $\mathcal{Q} \subseteq\left(\mathcal{Q}_{(i)}\right)^{(i)}$ for any $i \in \underline{n}$. Now suppose $X \notin \mathcal{Q}$. Since $\left(f_{11}^{1}+\cdots+f_{n n}^{1}\right) X=X \notin \mathcal{Q}$, there exists $k \in \underline{n}$ such that $f_{k k}^{1} X \notin \mathcal{Q}$. Furthermore from the fact that $\mathcal{Q}$ is 1 -prime, we can find $T \in \mathcal{M}_{n}(B, R)$ such that $f_{k k}^{1} X T f_{k k}^{1} X \notin \mathcal{Q}$. This implies $f_{k k}^{1} X T f_{k k}^{1} \notin \mathcal{Q}$. Note that $f_{k k}^{t}=f_{k k}^{1} X T f_{k k}^{1} \notin \mathcal{Q}$ where $t=\pi_{k} X T \bar{\epsilon}_{k}$. Apply the preceding lemma to obtain $X T \notin\left(\mathcal{Q}_{(k)}\right)^{(k)}$. So $X \notin\left(\mathcal{Q}_{(k)}\right)^{(k)}$. This yields $\left(\mathcal{Q}_{(k)}\right)^{(k)}=\mathcal{Q}$. The last part is now an immediate consequence.

Observe that in the above proposition if also $b_{j k}=b_{k j}=1$, then $\left(\mathcal{Q}_{(j)}\right)^{(j)}=\mathcal{Q}$. (See Proposition 2.6 and Lemma 2.15.) Theorems 3.1, 3.2, and 3.4 lead to our next results:

\section{Theorem 3.5.}

(1) $\operatorname{Spec}_{0}\left(\mathcal{M}_{n}(B, R)\right) \supseteq\left\{P^{(i)} \mid P \in \operatorname{Spec}_{0}(R)\right.$ for $\left.i \in \underline{n}\right\}$.

(2) $\operatorname{Spec}_{v}\left(\mathcal{M}_{n}(B, R)\right)=\left\{P^{(i)} \mid P \in \operatorname{Spec}_{v}(R)\right.$ for $\left.i \in \underline{n}\right\}$ for $v=1, e$.

Theorem 3.6. $\mathbb{P}_{\nu}\left(\mathcal{M}_{n}(B, R)\right)=\cap_{i=1}^{n}\left(\mathbb{P}_{\nu}(R)\right)^{(i)}$ for $\nu=1$ or $e$.

To end this section, we study the cardinalities of $\operatorname{Spec}_{\nu}\left(\mathcal{M}_{n}(B, R)\right)$ for $\nu=0,1, e$. But first we let $|W|$ be the cardinal of $W$ for any set $W$.

Definition 3.7 Let $\approx$ be a relation on $\underline{n}$ (with respect to $B$ ) defined via:

$$
i \approx j \text { if and only if } b_{i j}=b_{j i}=1 \text {. }
$$

Obviously, $\approx$ is an equivalence relation on $\underline{n}$. For convenience, denote by $\beta$ the number of the equivalence classes induced by $\approx$ on $\underline{n}$. For instance, if $B$ is an upper triangular matrix, then $i \approx j$ if and only if $i=j$. Hence $\beta=n$.

\section{Theorem 3.8.}

(1) $\left|\operatorname{Spec}_{0}\left(\mathcal{M}_{n}(B, R)\right)\right| \geq\left|\operatorname{Spec}_{0}(R)\right| \cdot \beta$.

(2) $\left|\operatorname{Spec}_{\nu}\left(\mathcal{M}_{n}(B, R)\right)\right|=\left|\operatorname{Spec}_{\nu}(R)\right| \cdot \beta$ for $\nu=1$ or $e$.

Proof. See Lemma 2.15 and Theorem 3.5.

\section{Concluding Remarks}

In [11], van der Walt and van Wyk defined the set $R^{n}(j, L)$ to be:

$\left\{\bar{u} \in R^{n} \mid u_{k}=0\right.$ if $b_{j k}=1$ and $b_{k j}=0$, and $u_{k} \in L$ if $\left.b_{j k}=b_{k j}=1\right\}$, where $L \subseteq R$ and $j \in \underline{n}$ (see also [12] and [13]). They investigated the set $\left(R^{n}(j, L)\right.$ : $\left.R^{n}(j, R)\right)$ and proved that:

$$
J_{2}\left(\mathcal{M}_{n}(B, R)\right)=\cap_{j=1}^{n}\left(R^{n}\left(j, J_{2}(R)\right): R^{n}(j, R)\right) .
$$

We show that $\left(R^{n}(j, L): R^{n}(j, R)\right)$ coincides with $L^{(j)}$. This implies that we obtain a description of, for example, $\mathbb{P}_{1}\left(\mathcal{M}_{n}(B, R)\right)$ analogous to the description of $J_{2}\left(\mathcal{M}_{n}(B, R)\right)$ mentioned above. Without loss of generality, assume $0 \in L$, otherwise both $\left(R^{n}(j, L)\right.$ : 
$\left.R^{n}(j, R)\right)$ and $L^{(j)}$ are empty. We need the following observations, the proofs are immediate from definitions:

(1) $R^{n}(j, L) \subseteq \amalg(j, L)$;

(2) $\Pi(R, j) \subseteq R^{n}(j, R)$;

(3) $R^{n}(j, L)=R^{n}(j, R) \cap \coprod(j, L)$.

Use (1) and (2) to obtain $\left(R^{n}(j, L): R^{n}(j, R)\right) \subseteq(\amalg(j, L): \Pi(R, j))=L^{(j)}$.

Assume $X \in L^{(j)}$. To complete the proof, we need to show that $X\left(R^{n}(j, R)\right) \subseteq$ $R^{n}(j, L)$. However, from part (3) and the fact that $R^{n}(j, R)$ is an $\mathcal{M}_{n}(B, R)$-ideal of $R^{n}$ [Corollary 3.6, 11], it suffices to show $X\left(R^{n}(j, R)\right) \subseteq \coprod(j, L)$. This is equivalent to showing that $\pi_{k} X \bar{u} \in L$ whenever $\bar{u} \in R^{n}(j, R)$ and $b_{j k}=1$. Assume $\bar{u}=\left(u_{1}, \cdots, u_{n}\right) \in$ $R^{n}(j, R)$ and $b_{j k}=1$. Furthermore if we could find an element $\bar{v}$ of $\Pi(R, j)$ such that $\bar{u} \sim_{k} \bar{v}$, then Lemma 2.3 (2) yields $X \bar{u} \sim_{k} X \bar{v}$ and so $\pi_{k} X \bar{u}=\pi_{k} X \bar{v} \in L$. (Since $X \in L^{(j)}$ and $\bar{v} \in \Pi(R, j)$, we have $X \bar{v} \in \coprod(j, L)$.)

Let $\bar{v}=\left(v_{1}, \cdots, v_{n}\right) \in R^{n}$ such that $v_{m}=u_{m}$ if $b_{m j}=b_{j m}=1$, and $v_{m}=0$ otherwise. Thus $\bar{v}$ is an element of $\Pi(R, j)$. Suppose $b_{k m}=1$. We then have $b_{j m}=1$ (since $b_{j k}=1$ ). Therefore there are two possible cases: $b_{m j}=1$ or $b_{m j}=0$. If $b_{m j}=1$, then $v_{m}=u_{m}$. If $b_{m j}=0$, then $v_{m}=0$ and $u_{m}=0$ (since $b_{j m}=1$ and $\left.b_{m j}=0\right)$. This implies $\bar{u} \sim_{k} \bar{v}$. We are done.

Veldsman [14] used an example (of a finite near-ring $R$ ) given by Meldrum and Meyer [8] to show that $\mathbb{P}_{0}\left(\mathcal{M}_{n}(R)\right)$ could be strictly contained in $\left(\mathbb{P}_{0}(R)\right)^{*}$. (Note that $\mathcal{M}_{n}(R)=\mathcal{M}_{n}(B, R)$ where $B=\left[b_{i j}\right]$ with $b_{i j}=1$ and $\left(\mathbb{P}_{0}(R)\right)^{*}=\left(\mathbb{P}_{0}(R)\right)^{(k)}$ for any $k \in \underline{n}$.) It would be interesting to determine the prime radical and all prime ideals of any matrix near-ring (or structural matrix near-ring).

The author thanks the referee for many helpful comments.

\section{References}

[1] G. L. Booth and N. J. Groenewald, "On primeness in matrix near-rings," Arch. Math., 56 (1991), 539-546.

[2] G. L. Booth, N. J. Groenewald, and S. Veldsman, "A Kurosh-Amitsur prime radical for near-rings," Comm. Alg., 18 (1990), 3111-3122.

[3] J. R. Clay, Nearrings: Geneses and Applications, Oxford Science Publications, Oxford, New York, Tokyo, 1992.

[4] N. J. Groenewald, "Different prime ideals in near-rings," Comm. Alg., 19 (1991), 2667-2675.

[5] H. E. Heatherly, "Matrix near-rings," J. London Math. Soc., 7 (1973), 355-356.

[6] S. Ligh, "A note on matrix near-rings," J. London Math. Soc., 11 (1975), 383-384.

[7] J. D. P. Meldrum, Near-Rings and Their Links with Groups, Pitman, Marshfield, M. A., 1985.

[8] - and J. H. Meyer, "Modules over matrix near-rings and the J0-radical," Monatsh. Math., 112 (1991), 125-139.

[9] - and A. P. J. van der Walt, "Matrix near-rings," Arch. Math. 47 (1986), 312-319.

[10] G. Pilz, Near-Rings, 2nd. edition, North-Holland, Amsterdam, 1983.

[11] A. P. J. van der Walt and L. van Wyk, "The $J_{2}$-radical in structural matrix near-rings," J. Algebra, 123 (1989), 248-261.

[12] L. van Wyk, "The 2-primitive ideals of structural matrix near-rings," Proc. Edinburgh Math. Soc., 34 (1991), 229-239. 
[13] —, "Maximal left ideals in structural matrix rings," Comm. Alg. 16 (1988), 399-419.

[14] S. Veldsman, "Special radicals and matrix near-rings," J. Austral. Math. Soc. (Series A), 52 (1992), 356-367.

Mathematics Department, University of Southwestern Louisiana, Lafayette, LA 70504, USA. 